Supporting Information for:

\title{
Molecular Mechanism for Allosteric Inhibition of Human Serotonin Transporter by Antidepressant Escitalopram
}

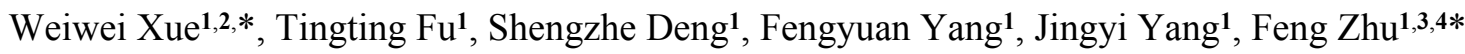

${ }^{1}$ Chongqing Key Laboratory of Natural Product Synthesis and Drug Research, Innovative Drug Research Center, School of Pharmaceutical Sciences, Chongqing University, Chongqing 401331, China

${ }^{2}$ Central Nervous System Drug Key Laboratory of Sichuan Province, Luzhou 646000, China

${ }^{3}$ College of Pharmaceutical Sciences, Zhejiang University, Hangzhou 310058, China

4 Innovation Institute for Artificial Intelligence in Medicine of Zhejiang University, Alibaba-Zhejiang University Joint Research Center of Future Digital Healthcare, Hangzhou 330110, China

*Corresponding Authors: Dr. Weiwei Xue, School of Pharmaceutical Sciences, Chongqing University, Chongqing 401331, China. Tel.: +86-023-6567-8450. Email: xueww@cqu.edu.cn; Prof. Feng Zhu, College of Pharmaceutical Sciences, Zhejiang University, Hangzhou 310058, China. Tel.: +86-571-8820-8444.

Email: zhufeng@zju.edu.cn 
Table S1. The average RMSD values of the designed systems during the cMD simulations.

\begin{tabular}{llllll}
\hline \multirow{2}{*}{ Systems } & \multicolumn{7}{c}{ Average RMSD $(\AA)$} & \\
\cline { 2 - 6 } & Protein $^{a}$ & S1 site $^{b}$ & Ligand_S1 $^{c}$ & S2 site $^{b}$ & Ligand_S2 $^{c}$ \\
\hline Escitalopram_S1 & 2.66 & 0.67 & 0.71 & - & - \\
\hline Escitalopram_S2 & 2.71 & - & - & 1.40 & 1.75 \\
\hline Escitalopram_S1 (S2 bound ) & 1.99 & 0.59 & 0.69 & 0.99 & 1.75 \\
\hline Paroxetine_S1 & 2.38 & 0.75 & 0.84 & - & - \\
\hline
\end{tabular}

${ }^{a}$ The whole protein backbone atom.

${ }^{b}$ The backbone atom of residues around $5 \AA$ of ligand.

${ }^{c}$ The heavy atom of the ligand. 
Table S2. The total and decomposed terms $(\mathrm{kcal} / \mathrm{mol})$ of the free energies for the drugs binding to SERT.

\begin{tabular}{|c|c|c|c|c|c|c|c|}
\hline Drugs & Targets & & $\Delta G_{\text {calc }}^{a}$ & $\Delta E_{\text {ele }^{b}}^{b}$ & $\Delta E_{\mathrm{vdW}}{ }^{c}$ & $\Delta G_{\mathrm{pol}}{ }^{d}$ & $\Delta G_{\text {nonpol }^{e}}$ \\
\hline \multirow{4}{*}{ Escitalopram } & $\operatorname{SERT}(\mathrm{S} 1)^{f}$ & & -48.85 & -38.73 & -47.43 & 43.46 & -6.16 \\
\hline & SERT (S2) ${ }^{g}$ & & -38.57 & -39.18 & -38.54 & 44.34 & -5.19 \\
\hline & \multirow{2}{*}{ SERT $(\mathrm{S} 1-\mathrm{S} 2)^{h}$} & $\mathrm{~S} 1$ & -50.38 & -29.01 & -48.67 & 33.48 & -6.18 \\
\hline & & $\mathrm{S} 2$ & -36.91 & -24.70 & -37.19 & 29.99 & -5.01 \\
\hline Paroxetine & $\operatorname{SERT}(\mathrm{S} 1)^{f}$ & & -49.97 & -36.55 & -46.75 & 33.33 & -5.96 \\
\hline
\end{tabular}

${ }^{a}$ The binding free energy calculated by MM/GBSA method $\left(\Delta G_{\text {calc }}=\Delta E_{\text {ele }}+\Delta E_{\mathrm{vdW}}+\Delta G_{\mathrm{pol}}\right.$ $\left.+\Delta G_{\text {nonpol}}\right)$.

${ }^{b}$ The electrostatic interaction energy in gas phase.

${ }^{c}$ The van der Waals interaction energy in gas phase.

${ }^{d}$ The free energy of polar solvation.

${ }^{e}$ The free energy of nonpolar solvation.

${ }^{f}$ Drug binding in the $\mathrm{S} 1$ site of SERT.

${ }^{g}$ Drug binding in the S2 site of SERT.

${ }^{h}$ Drug simultaneously binding in the S1 and S2 sites of SERT. 
Table S3. The energies $(\mathrm{kcal} / \mathrm{mol})$ of important residues contributed to drugs binding to SERT.

\begin{tabular}{|c|c|c|c|c|c|}
\hline Residues & $\begin{array}{l}\text { Escitalopram_S1 } \\
\text { (S2 bound) }\end{array}$ & Escitalopram_S1 & Residues & $\begin{array}{l}\text { Escitalopram_S2 } \\
\text { (S1 bound) }\end{array}$ & Escitalopram_S2 \\
\hline Tyr95 & -3.59 & -3.53 & Asp98 & -1.15 & -0.11 \\
\hline Asp98 & -1.01 & -1.05 & Ala331 & -1.06 & -1.05 \\
\hline Ile172 & -3.01 & -3.12 & Gln332 & -1.29 & -1.25 \\
\hline Tyr176 & -2.12 & -2.09 & Ser336 & -1.59 & -0.5 \\
\hline Phe335 & -1.18 & -1.21 & Glu493 & -1.34 & -0.96 \\
\hline Ser336 & -0.98 & -1.11 & Glu494 & -1.45 & -1.42 \\
\hline Thr439 & -1.32 & -1.43 & Phe556 & -0.55 & -0.55 \\
\hline Gly442 & -1.37 & -1.25 & & & \\
\hline Leu443 & -0.66 & -0.7 & & & \\
\hline Val501 & -0.87 & -0.56 & & & \\
\hline
\end{tabular}



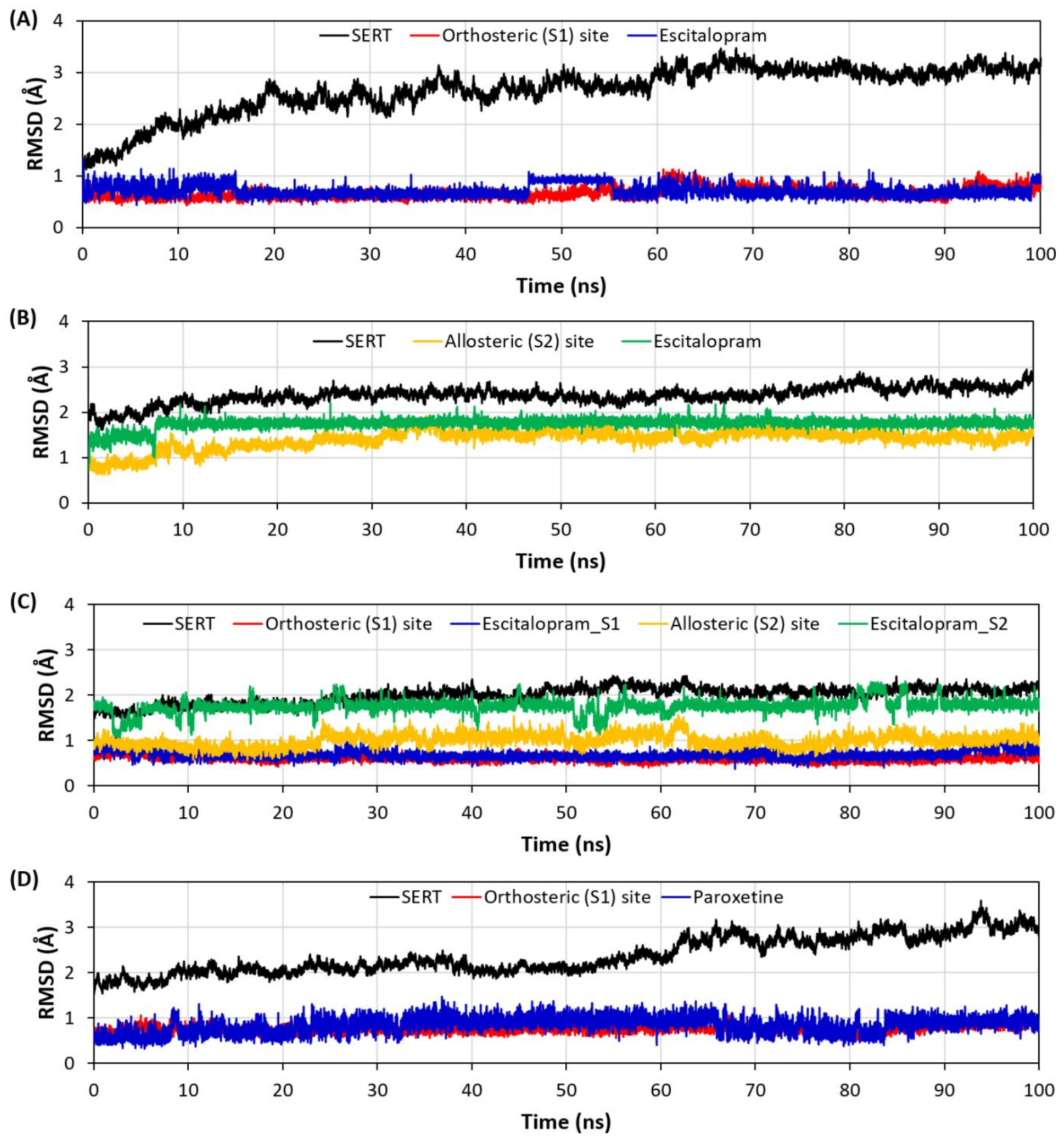

Figure S1. The time evolution of the RMSD of backbone atoms of SERT and heavy atoms of drugs for each system with respective to the crystal coordinates. 


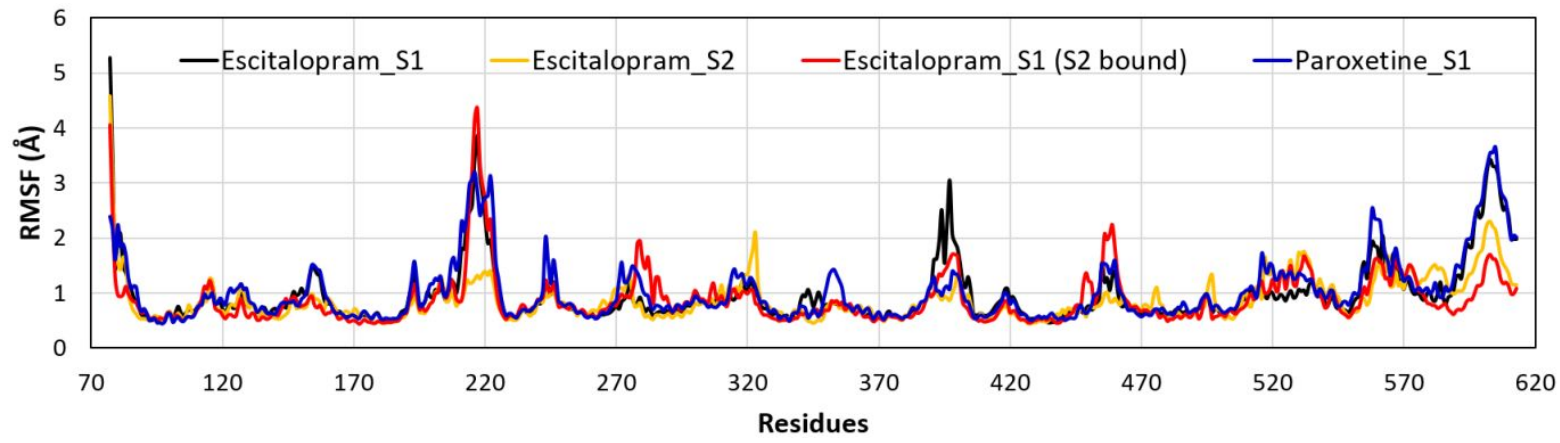

Figure S2. Per-residue RMSF of SERT for each system calculated from cMD simulations. 


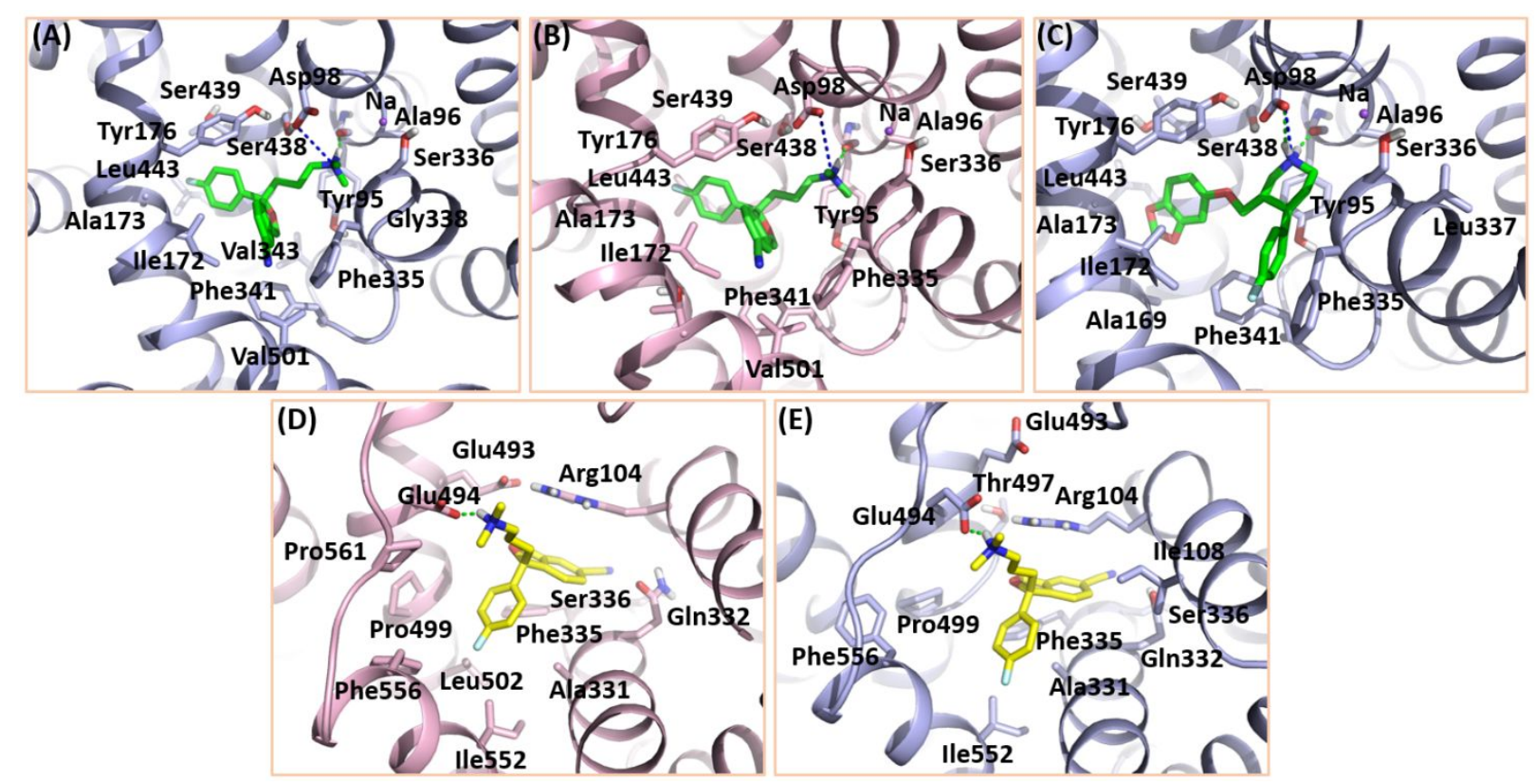

Figure S3. The representative snapshots of the drugs binding in S1 and (or) S2 site of SERT derived from the equilibrated trajectories. (A) escitalopram binding in S1 site, (B) escitalopram binding in $\mathrm{S} 1$ site (S2 bound), (C) paroxetine binding in S1 site, (D) escitalopram binding in $\mathrm{S} 2$ site (S1 bound) and (E) escitalopram binding in S2 site. The protein and ligands were displayed as cartoon and stick representation, respectively. Salt bridge and hydrogen bond were shown in blue and green dashed lines, respectively. 


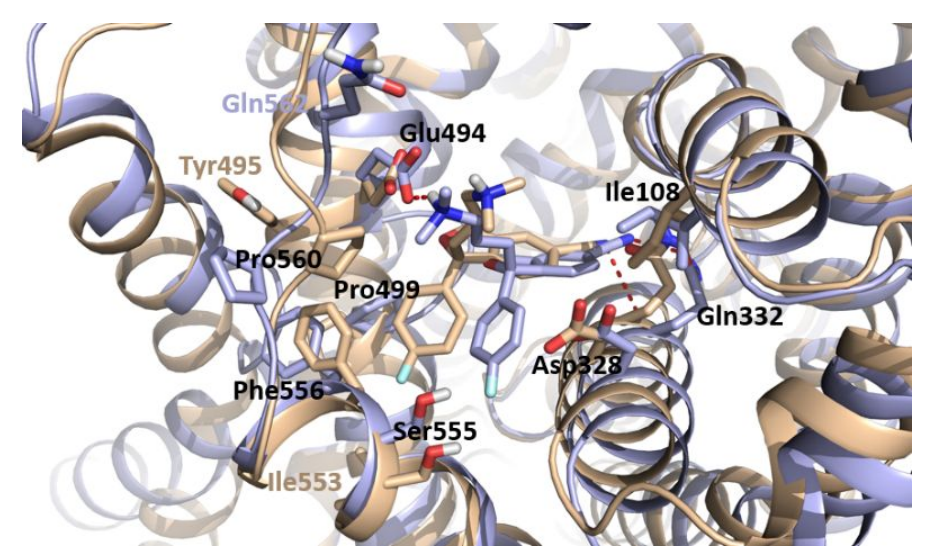

Figure S4. Structural alignment of escitalopram binding at SERT S2 site before (wheat) and after (light blue) MD simulation. The protein and ligands were displayed as cartoon and stick representation, respectively. Hydrogen bonds were shown in red dashed lines. 


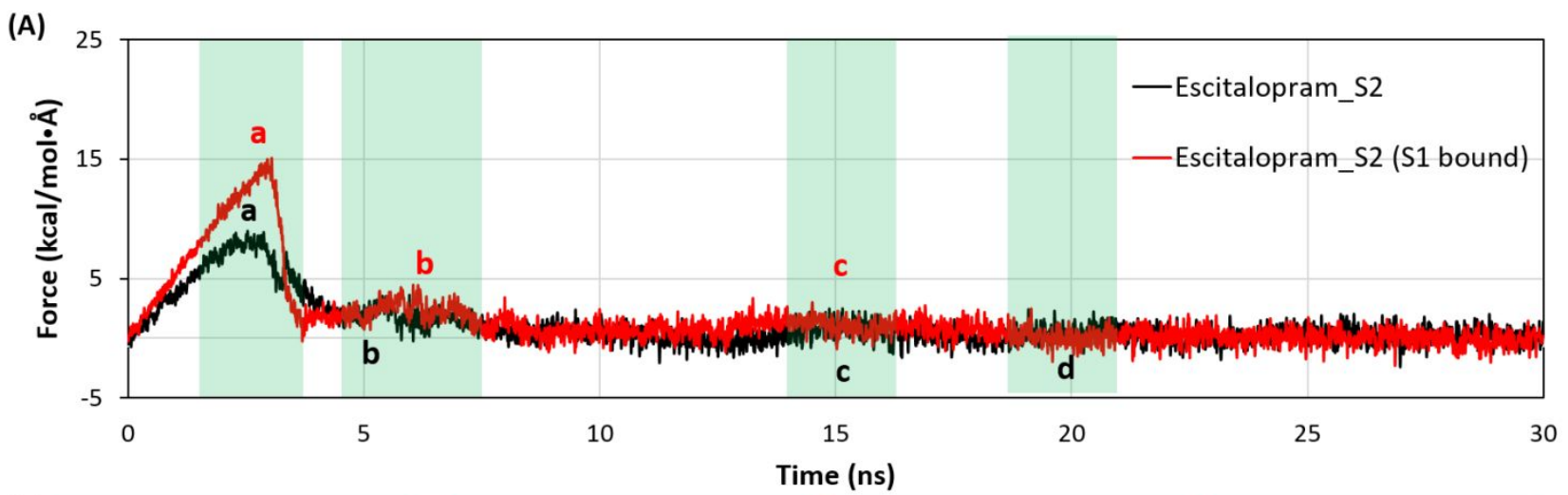

(B)
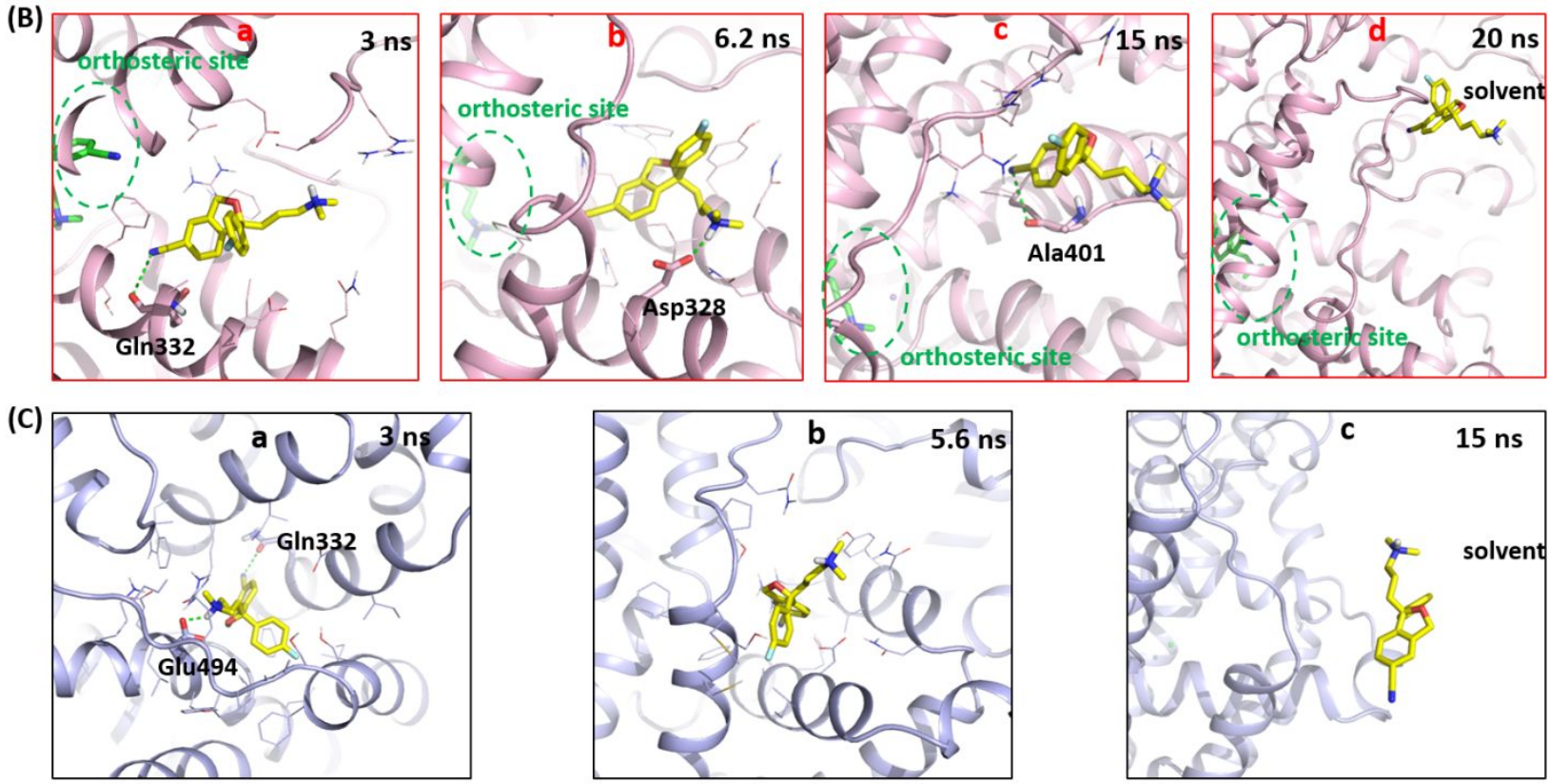

Figure S5. The applied force and intermediate snapshots of each system during the simulation.

(A) Force vs Time of escitalopram dissociated from S2 site of SERT during SMD simulations. The drugs were pulled at $0.001 \AA /$ ps using a time step of 2 fs. (B-C) The extracted snapshots for the relative positions of drug and transporter along the unbinding pathways for escitalopram (S1 bound) (red boxes a-d) and escitalopram (black boxes a-c). The SERT and drugs were displayed in cartoon and stick representation, respectively. The salt bridge and hydrogen bond were shown in blue and green dashed lines, respectively. 